\title{
Effects of Salvia miltiorrhiza extract on lung adenocarcinoma
}

\author{
HUIXIANG TIAN ${ }^{1,2}$, YUEQIN LI $^{3}$, JIE MEI $^{2}$, LEI CAO ${ }^{1,2}$, JIYE YIN $^{2}$, \\ ZHAOQIAN LIU ${ }^{2}$, JUAN CHEN ${ }^{1}$ and XIANGPING LI ${ }^{1,2}$
}

\author{
Departments of ${ }^{1}$ Pharmacy, ${ }^{2}$ Clinical Pharmacology and ${ }^{3}$ Integrated Traditional Chinese and Western Medicine, \\ Xiangya Hospital, Central South University, Changsha, Hunan 410008, P.R. China
}

Received June 17, 2020; Accepted April 1, 2021

DOI: $10.3892 / \mathrm{etm} .2021 .10226$

\begin{abstract}
Lung adenocarcinoma is the most common subtype of non-small cell lung carcinoma. Tanshinone I is an important fat-soluble component in the extract of Salvia miltiorrhiza that has been reported to inhibit lung adenocarcinoma cell proliferation. However, no studies have clearly demonstrated changes in lung adenocarcinoma gene expression and signaling pathway enrichment following Tanshinone I treatment. And it remains unclear whether salvianolate has an effect on lung adenocarcinoma. The present study downloaded the GSE9315 dataset from the Gene Expression Omnibus database to identify differentially expressed genes (DEGs) and the underlying signaling pathways involved after Tanshinone I administration in the lung adenocarcinoma cell line CL1-5. The results revealed that there were 28 and 102 DEGs in the low dosage group (0.01 and $0.10 \mu \mathrm{g} / \mathrm{ml}$ Tanshinone I) and medium dosage groups ( 1 and $10 \mu \mathrm{g} / \mathrm{ml}$ Tanshinone I), respectively. In the low dosage group, DEGs were mainly enriched in 'positive regulation of T-helper cell differentiation' and 'protein complex'. In the medium dosage group, 102 DEGs were enriched in 'MAPK cascade' and 'extracellular exosome'. Kyoto Encyclopedia of Genes and Genomes pathway analysis demonstrated enrichment of both groups in the PI3K-Akt signaling pathway. Furthermore, there were nine overlapping DEGs [ADP ribosylation factor-interacting protein 2, chemokine (C-X-C motif) ligand 6, SH2 domain-containing adaptor protein $\mathrm{B}$, Src homology 2 domain-containing transforming protein1, collagen type VI $\alpha 1$ chain, elastin, integrin subunit $\alpha$, endoplasmic reticulum mannosyl-oligosaccharide 1,2- $\alpha$-mannosidase and sterile $\alpha$ motif domain-containing 9 like] between the two groups, which serve to be potential targets for the treatment of lung adenocarcinoma. The present study also investigated the possible effects of salvianolate on lung adenocarcinoma in vivo using nude mouse xenograft
\end{abstract}

Correspondence to: Professor Xiangping Li, Department of Pharmacy, Xiangya Hospital, Central South University, 87 Xiangya Road, Changsha, Hunan 410008, P.R. China

E-mail: xylxping@126.com

Key words: tanshinone I, salvianolate, lung adenocarcinoma, differentially expressed genes, anticancer effect models injected with the A549 cell line. The data revealed that salvianolate not only suppressed lung adenocarcinoma tumor growth of in nude mice, but also downregulated the expression levels of ATP7A and ATP7B, which are important proteins in the tumorigenesis and chemotherapy of lung adenocarcinoma. The present study provided evidence for the potential use of Salvia miltiorrhiza extract for treating lung adenocarcinomas in the clinic.

\section{Introduction}

Lung cancer is a type of malignant tumor that continues to be the leading cause of cancer-associated mortality worldwide (1). The estimated number of new cases and deaths from lung cancer worldwide in 2020 were 2,206,771 and 1,796,144, respectively (2). As a subtype of lung cancer, lung adenocarcinoma has the highest incidence of all types of lung cancer (3). Surgery, chemotherapy, radiotherapy, targeted therapies and combined therapies are the therapeutic strategies that are currently available (4). However, poor patient responses to therapy, individual differences, adverse side effects and resistance to chemotherapeutic agents mean that clinical difficulties remain for the treatment of lung adenocarcinoma (5-7).

Traditional Chinese medicine (TCM) has been reported to have anti-cancer effects in lung adenocarcinoma, osteosarcoma and other types of cancer by regulating the tumor microenvironment and enhancing host immune responses (8-10). In particular, the red sage Salvia miltiorrhiza (Danshen) has been reported to improve survival rate for patients with colon (11) and breast cancer (12). The chemical composition of Salvia miltiorrhiza includes two categories (13): i) Fat-soluble tanshinone compounds, which are mainly comprised of Tanshinone I and Tanshinone IIA; and ii) water-soluble phenolic compounds, consisting mainly of salvianolate. Tung et al (14) found Tanshinone I to inhibit the proliferation of lung adenocarcinoma cell lines A549, CL1-0 and CL1-5, which was in turn more effective compared with Tanshinone II. However, to the best of our knowledge, no studies have clearly demonstrated changes in gene expression and pathway enrichment following Tanshinone I administration on lung adenocarcinoma. Furthermore, as a major water-soluble component of Salvia miltiorrhiza extract, it remains currently unclear whether salvianolate has an effect on lung adenocarcinoma.

ATPase copper transporting $\alpha$ and $\beta$ (ATP7A and ATP7B) are heavy metal transporting P-type ATPases that function as 
copper efflux transporters to maintain cellular copper homeostasis (15). It has been previously shown that ATP7A/7B has effects on tumorigenesis (16), tumor cell differentiation (17) and platinum-based chemotherapy response $(18,19)$ in breast, lung and ovarian cancer. In addition, previous studies suggest that ATP7A/7B are considered to be potential targets for the treatment of non-small cell lung cancer (NSCLC) $(18,20,21)$. Therefore, present study investigated the effect of ATP7A/7B expression following salvianolate treatment.

Numerous profiles in carcinogenesis and cancer progression have been screened following the development of microarrays and high-throughput sequencing. The present study analyzed the gene expression profile matrix file (GSE9315) (22) using a series of bioinformatics tools to identify hub genes and key pathways that are affected by Tanshinone I administration on lung adenocarcinoma. The effects of salvianolate were then investigated using a xenograft nude mouse model before the potential underlying mechanisms of action were assessed. The present study identified the underlying mechanisms of action and potential targets of the Salvia miltiorrhiza extract for the treatment of lung adenocarcinoma.

\section{Materials and methods}

Data source. The gene expression profile matrix file (GSE9315) was downloaded from the Gene Expression Omnibus (GEO) database (https://www.ncbi.nlm.nih.gov/geo/). GSE9315 contained five different concentrations of Tanshinone I $(0.00$, $0.01,0.10,1.00$ and $10.00 \mu \mathrm{g} / \mathrm{ml})$ in macrophage-conditioned medium (CM) and a control condition without CM in the lung adenocarcinoma cell line CL1-5. Its platform used was GPL5968 (NCHU_M\&A_human 1152 cDNACHIP).

Identification of differentially expressed genes (DEGs) following different doses of Tanshinone I. GEO2R (https://www. ncbi.nlm.nih.gov/geo/geo2r/) is an interactive web tool that was used to analyze the raw data and identify DEGs in the GSE9315 dataset. GSE9315 was divided into the following three groups: i) Control group ( $0 \mu \mathrm{g} / \mathrm{ml}$ in CM); ii) low dosage group (0.01 and $0.1 \mu \mathrm{g} / \mathrm{ml}$ in CM); and iii) medium dosage group (1 and $10 \mu \mathrm{g} / \mathrm{ml}$ in CM) (22). In the present study, |log fold change (FC) $\mid>1$ was used as the cut-off criteria to identify DEGs. $\mathrm{P}<0.05$ was not used as a criterion for screening DEG since there was only one sample in the control group, which was not suitable for statistical analysis. A Venn diagram was used to identify overlapping DEGs in the low and medium dosage groups.

Gene Ontology (GO) and Kyoto Encyclopedia of Genes and Genomes (KEGG) enrichment analyses of DEGs. The Database for Annotation, Visualization and Integrated Discovery (DAVID; version 6.8; https://david.ncifcrf. gov/) (23) was used to perform the GO and KEGG pathway enrichment analyses. The results were visualized using ggplot2 package in R Studio (version 1.1.453; The R Foundation) (24). $\mathrm{P}<0.05$ was considered to indicate a statistically significant difference.

Protein-protein interaction (PPI) network construction and module analysis. The Search Tool for the Retrieval of Interacting Gene (version 11.0; https://string-db.org/cgi/input.pl) database (25) was used to construct a functional PPI network of the DEGs. An interaction score of 0.4 was regarded as the cut-off criterion in the present study. The Molecular Complex Detection (MCODE) in Cytoscape software (version 3.6.0; https://cytoscape.org/) (26) was then used to find closely connected regions in the PPI network. The selection criteria were as follows: i) Degree Cut-off, 2; ii) node score cut-off, 0.2; iii) K-Core, 2; and iv) max depth, 100.

Cell culture. The human lung adenocarcinoma cell line A549 (Changsha Nanke Biotechnology Co., Ltd.; http://www.nkbio. cn) was cultured in RPMI-1640 medium (Gibco; Thermo Fisher Scientific, Inc.) supplemented with 10\% FBS (Gibco; Thermo Fisher Scientific, Inc.) at $37^{\circ} \mathrm{C}$ with $5 \% \mathrm{CO}_{2}$.

Establishment and drug administration in animal models. The present study was performed according to the guidelines of the Ethics Committee of Institutional Animal Care and Use in Central South University (27) and approved by the Medical Ethics Committee of Xiangya Hospital, Central South University (approval no. 2017121170; Changsha, China). A total of 30,6-week-old nude mice (weight range, 16-18 g; sex, 1:1 ratio of males and females) purchased from Hunan SJA Laboratory Animal Co., Ltd, were kept under specific-pathogen free conditions (sterile laminar flow chamber, room temperature $21-25^{\circ} \mathrm{C}$; humidity of $60-70 \%$; $12 \mathrm{~h}$ light/dark cycle) with access to food and drinking water ad libitum at the Department of Laboratory Animals, Central South University. A549 cells in the logarithmic growth phase were harvested and resuspended in PBS at a density of $1 \times 10^{6} / \mathrm{ml}$. After being anesthetized by isoflurane inhalation $(1.5 \%)$, the nude mice were injected subcutaneously into the left armpit in a cell suspension of $0.2 \mathrm{ml}\left(2 \times 10^{5}\right.$ cells per mouse). The next day, the tumor volume of nude mice was measured, which was regarded as the first day after injection (day 1). The tumor volume was measured once every 3 days for 16 days (day 1, 4, 7, 10, 13 and 16). When the tumors became visible (day 4), 10, 20 and $50 \mathrm{mg} / \mathrm{kg}$ salvianolate (Green Valley, Inc.) and normal saline (Sichuan Kelun Pharmaceutical Co., Ltd.) were injected into the tumor-adjacent tissue once every 3 days (day 4, 7, 10 and 13). Mice were sacrificed by cervical dislocation on day 16 following the final treatment of salvianolate and normal saline before the tumor tissues were collected for further research. The tumor volume was measured using the following equation: $\mathrm{V}\left(\mathrm{mm}^{3}\right)=\mathrm{a} \times \mathrm{b}^{2} \mathrm{x} 0.52$, where ' $\mathrm{a}$ ' and ' $b$ ' were the longest and shortest diameters of the tumor. The tumor inhibition rate was calculated using the following equation: Inhibition rate $(\%)=\left(1-\mathrm{V}_{\text {salvianolate }} / \mathrm{V}_{\text {mean-control }}\right) \times 100 \%$. Lack of breathing or no basic vital signs were evaluated to ensure death after cervical dislocation. In the present study, 'The animal exhibiting depression and hypothermia without anesthesia or sedation (dying)' was regarded as the criterion for euthanizing the animals.

Hematoxylin and eosin $(H \& E)$ staining. The tumor tissues were fixed in $4 \%$ paraformaldehyde for $24 \mathrm{~h}$ at room temperature and embedded in paraffin. Tissue samples were then cut into $3-\mu \mathrm{m}$-thick slices and the slices were heated at $60^{\circ} \mathrm{C}$ for $1.5 \mathrm{~h}$. The slices were deparaffinized in xylene and rehydrated in graded ethanol solutions (100, 95, 80 and 70\% ethanol 
Table I. Differentially expressed genes identified in the different dosage groups.

Groups Gene symbol

Low dosage

Medium

dosage
TMEM41B, EVI1, CD9, GNG11, IFNAR2, RPS27L, PTGS2, ITGA2, CTNNB1, MYB, ID4, CAV2, MTHFS, CDKN1B, S100A8, IL18, MMP1, AOF1, MMP7, MAN1B1, SHB, ELN, SHC1, COL6A1, ACTG2, ARFIP2, CXCL6, SAMD9L

MAN1B1, ELN, NRP2, CDK9, NR1D1, NME3, PPIL2, FXN, SYT1, PCDHGC3, CACNA2D2, TMEM115, EIF4E2, SAMD9L, MTA1, ARFIP2, HYAL1, NOS3, MAPK1, C9orf164, SLC25A1, CFB, PAFAH1B3, CDK2AP2, TXLNA, FLI1, RBBP4, NBL1, RNF4, NR5A2, ERBB2, NCSTN, HLF, MARS, AK1, NAPA, SHC1, NEFL, MEN1, CD44, FGB, EIF3S4, TPM1, EPHX1, TIMP2, CSNK2B, TP53, CTSD, MUC1, EFNA1, FRAP1, RAB8A, ARFIP2, HMGCL, KRAS, GRN, ERBB2, CALM3, BAX, SLC25A38, FCGRT, ANXA4, BMP1, PLAU, RNF167, MYH9, ISG20, IGBP1, EMP2, NR4A1, ARMET, STAT1, LMAN2L, ANGPT1, MRPL23, TLR2, MAP2K3, SEPHS2, BCAT2, STC1, MRPL37, CD44, COL6A1, COL4A2, LAMB1, CXCL6, TM7SF2, DECR2, PPP2R5B, CKS1B, SHB, CCND3, SMAD2, JUNB, ITGA2, GNB1, IFITM1, EIF3S6, PITPNA, CHD8, CTDSP2, FDFT1 for $5 \mathrm{~min}$ at each concentration) at room temperature. After washing with water, slices were stained with hematoxylin for $2 \mathrm{~min}$, rinsed with water, incubated in $0.5 \% \mathrm{HCl}$ ethanol for 1-3 sec, rinsed with water and stained with eosin for $3 \mathrm{~min}$, all at room temperature. Then the slices were dehydrated using $70,80,95$ and $100 \%$ gradient ethanol and xylene for $5 \mathrm{~min}$ at room temperature, and sealed with neutral gum. The nuclei were blue-violet in color, whilst the cytoplasm and intercellular matrix were red under light microscopy when the histological changes were assessed (magnification, x400).

Immunohistochemistry (IHC). Paraffin-embedded tumor tissues, as aforementioned, were cut into 4- $\mu \mathrm{m}$-thick slices. Slices were dewaxed using xylene, rehydrated in an descending ethanol gradient and treated with $3 \%$ hydrogen peroxide for $10 \mathrm{~min}$ at room temperature to remove endogenous oxidases. The slices were then repaired using a citrate repairing solution ( $0.01 \mathrm{~mol} / \mathrm{l}$; pH 6.0; cat. no. C1010; Beijing Solarbio Science \& Technology Co., Ltd.), heated at $100^{\circ} \mathrm{C}$ for $15 \mathrm{~min}$ for antigen retrieval and washed for $3 \mathrm{~min}$ with PBS (0.01 mol/1, pH 7.2) after air cooling. Subsequently, each slice was blocked with 5\% goat serum (cat. no. SL038; Beijing Solarbio Science \& Technology Co., Ltd.) for $10 \mathrm{~min}$ at room temperature and incubated with primary antibodies against ATP7A (1:100; cat. no. NBP2-59376; Novus Biologicals, LLC.) or ATP7B (1:100; cat. no. ab133731; Abcam) overnight at $4^{\circ} \mathrm{C}$. After three washes with PBS, the slices were incubated with rabbit anti-mouse and goat anti-rabbit IgG horseradish peroxidase-conjugated secondary antibodies (both 1:1,000; cat. nos. ab6721 and ab6728, respectively; Abcam) for $2 \mathrm{~h}$ at room temperature. The sections were washed three times with PBS and incubated with streptavidin-peroxidase solution for $10 \mathrm{~min}$ at room temperature. Finally, DAB (Fuzhou Maixin Biotech Co., Ltd.) was used for visualization and hematoxylin for counterstaining, followed by dehydration with ethanol gradient $(70,80,90,95$ and $100 \%)$ followed by xylene and mounting with neutral gum. After visualizing, images of sections were captured using a light microscope (magnification, x400) and analyzed using Image-Pro Plus 6.0 software (Media Cybernetics, Inc.)
Statistical analysis. All data were processed using SPSS 19.0 statistical software (IBM Corp.). One-way ANOVA with Bonferroni's correction was used for the comparison between the various groups. Data are presented as the mean \pm SD. $\mathrm{P}<0.05$ was considered to indicate a statistically significant difference. The number of repeats in each group is $\geq 3$.

\section{Results}

Identification of DEGs after low or medium dosage treatment of Tanshinone I. Based on the aforementioned threshold ( $\log \mathrm{FCl}>1$ ), 28 and 102 DEGs were identified in the low dosage group and the medium dosage groups, respectively (Table I). Notably, there were nine overlapping DEGs in the low and medium dosage groups (Fig. 1A), which were integrin subunit $\alpha$ (ITGA2), endoplasmic reticulum mannosyl-oligosaccharide 1,2- $\alpha$-mannosidase (MAN1B1), $\mathrm{SH} 2$ domain containing adaptor protein B (SHB), elastin (ELN), Src homology 2 domain-containing) transforming protein 1 (SHC1), collagen type VI $\alpha 1$ chain (COL6A1), ADP ribosylation factor interacting protein 2 (ARFIP2), chemokine (C-X-C motif) ligand 6 (CXCL6) and sterile $\alpha$ motif domain containing 9 like (SAMD9L). The visualized heatmaps of the DEGs in the individualized different dosage groups are presented in Fig. 1B and C, which showed that some genes were upregulated (such as CD9, IFNAR2 and PTGS2) and others were downregulated (such as MAPK1, SLC25A1 and KRAS). In the heatmaps, red and green represent upregulation and downregulation of gene expression, respectively.

Analysis of overlapping DEGs in the low and medium dosage groups. There were nine overlapping DEGs in the low and medium dosage groups, which were ITGA2, MAN1B1, SHB, ELN, SHC1, COL6A1, ARFIP2, CXCL6 and SAMD9L. The visualization results of gene expression were displayed using a heatmap (Fig. 1D). Among these nine genes, eight were downregulated after four different dosages of Tanshinone I were administrated (shown as green in the heatmap), compared with the control group. Notably, the expression of these eight genes was not dose-dependent. Only one of the nine overlapping 


\section{A}

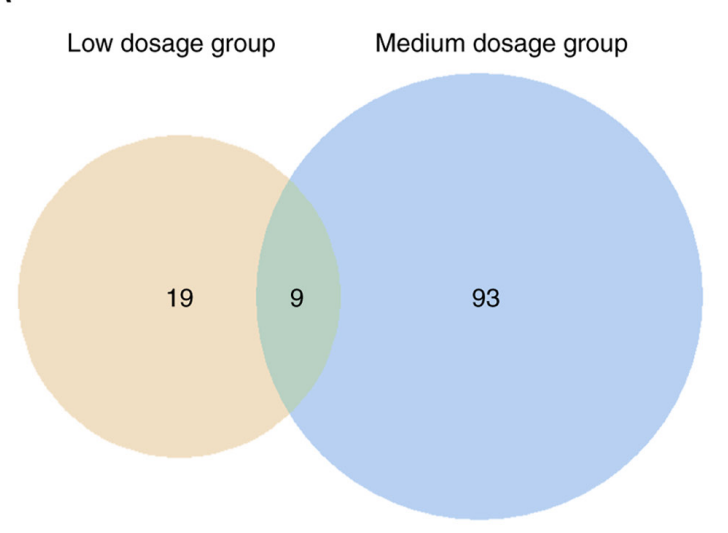

C

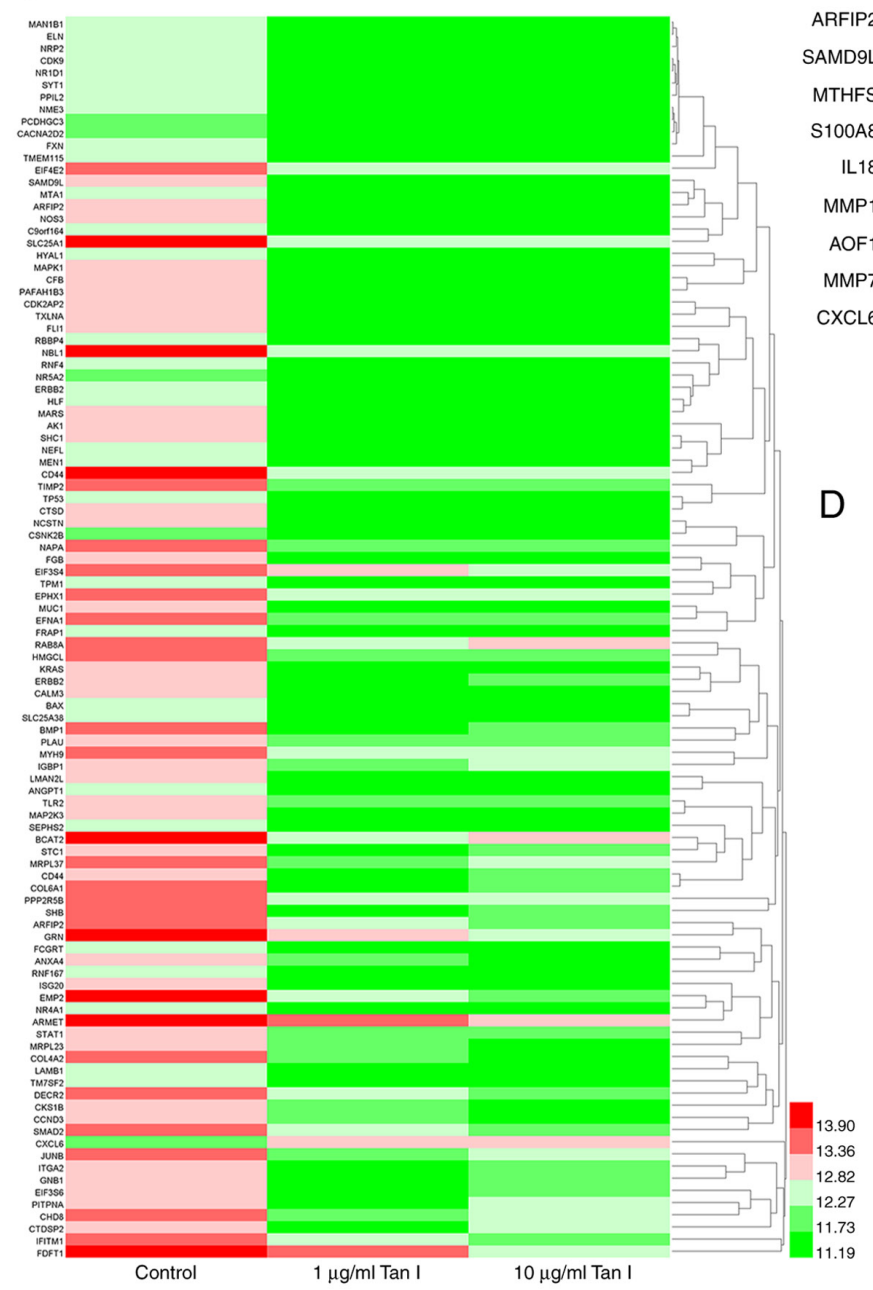

B
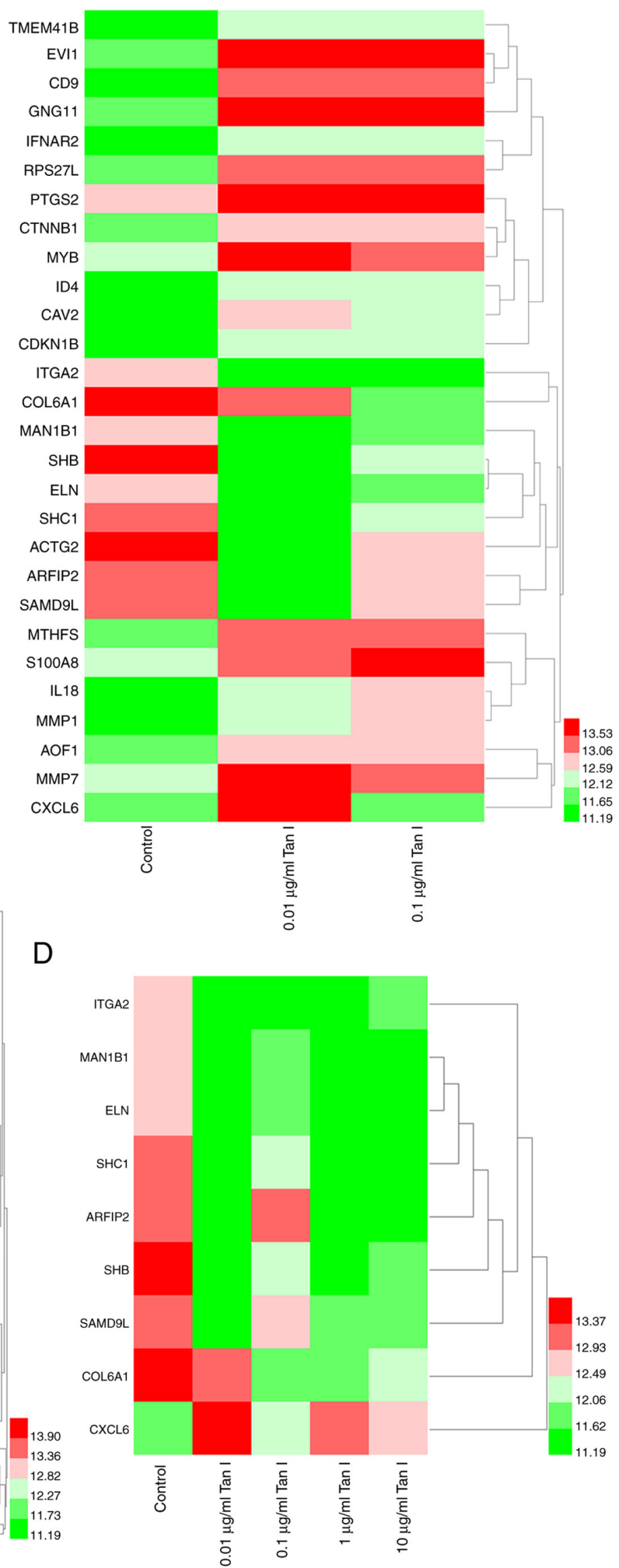

Figure 1. Venn diagram and heatmaps of DEGs in the different groups. (A) Venn diagram shows that there were nine overlapping DEGs in the low and middle dosage group, which contains 19 and 93 DEGs, respectively. Heatmap of DEGs (B) in the low dosage and (C) the medium dosage group. (D) Heatmap of the overlapping DEGs in the low and medium dosage groups. Red and green represent up- and downregulation of gene expression, respectively. DEGs, differentially expressed genes; Tan I, tanshinone I.

genes (CXCL6) was upregulated following Tanshinone I administration (shown as red in the heatmap). The GO functional enrichment and KEGG pathway analyses of each gene are presented in Table II, helping us understand the functional enrichment of each gene. For example, SHC1 was enriched in 'epidermal growth factor receptor (ErbB) signaling pathway' and COL6A1 was enriched in 'PI3K-Akt signaling pathway', indicating that Tanshinone I may treat lung adenocarcinoma by upregulating or downregulating these genes and their enriched pathways. 

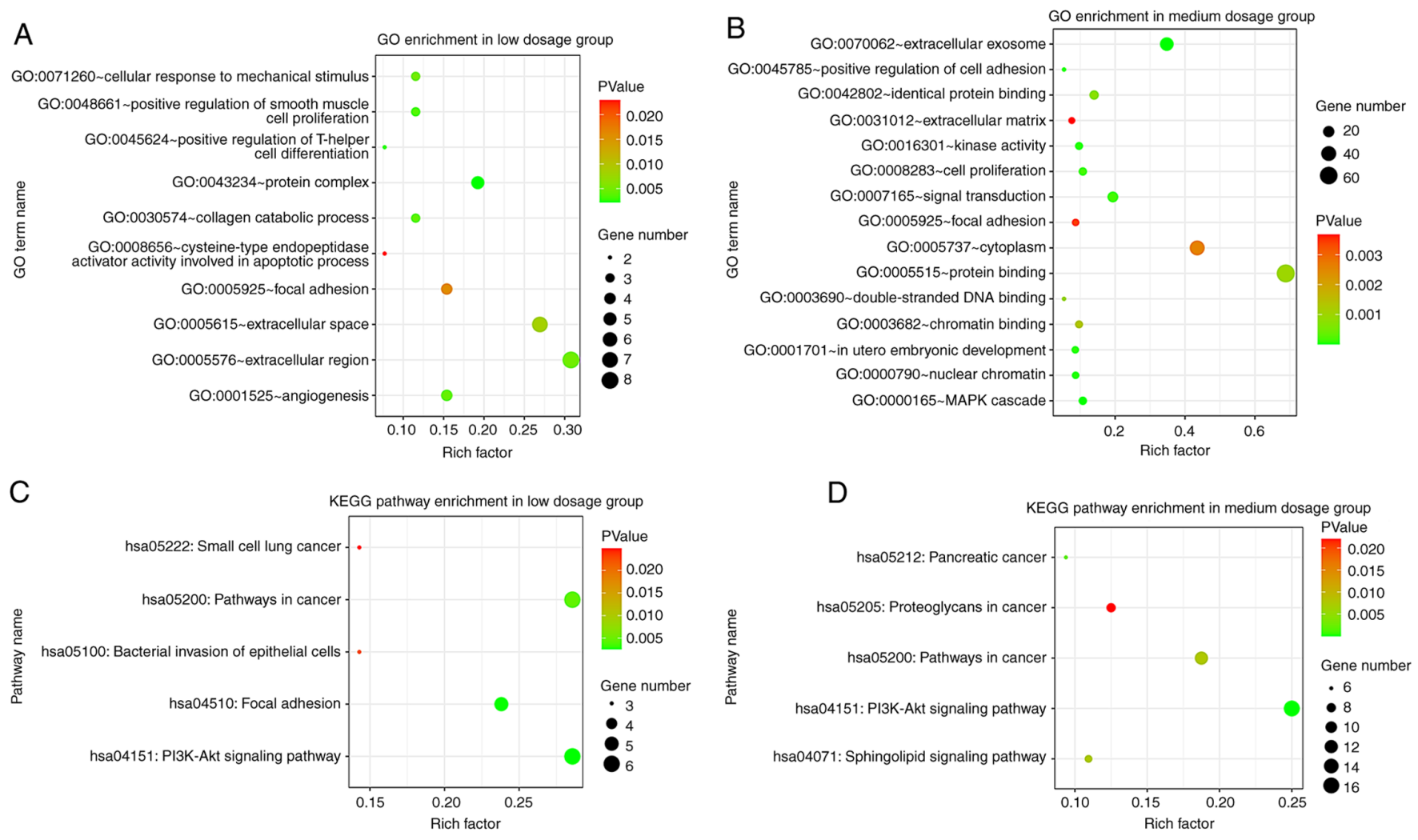

Figure 2. GO and KEGG pathway enrichment analysis in the low and medium dosage groups. GO enrichment analysis in the (A) low dosage and (B) medium dosage groups, and KEGG pathway enrichment analysis in the (C) low dosage and (D) medium dosage groups were performed using Database for Annotation, Visualization and Integrated Discovery, before being visualized using RStudio. GO, Gene Ontology; KEGG, Kyoto Encyclopedia of Genes and Genomes.

GO functional enrichment analysis of DEGs. Significant terms in the GO enrichment analysis are presented in Fig. 2A and B. The DEGs in the low dosage group (Fig. 2A) were particularly enriched in the biological processes (BP) including, "positive regulation of T-helper cell differentiation', 'positive regulation of smooth muscle cell proliferation', 'collagen catabolic process', 'angiogenesis' and 'cellular response to mechanical stimulus'. As for the cellular components (CC), the genes were enriched in 'protein complex', 'extracellular region', 'extracellular space' and 'focal adhesion'. The molecular function (MF) results showed that DEGs were most enriched in 'cysteine-type endopeptidase activator activity involved in apoptotic process'.

In the medium dosage group (Fig. 2B), BP genes were primarily enriched in the 'MAPK cascade', 'in utero embryonic development', 'positive regulation of cell adhesion', 'signal transduction' and 'cell proliferation'. For CC, the DEGs were mainly enriched in 'extracellular exosome', 'nuclear chromatin', 'cytoplasm', 'focal adhesion' and 'extracellular matrix'. MF genes were predominantly enriched in 'kinase activity', 'identical protein binding', 'protein binding', 'double-stranded DNA binding' and 'chromatin binding'.

KEGG pathway analysis of DEGs. The KEGG pathway enrichment analysis suggested that the DEGs in the low dosage group were mainly enriched in 'PI3K-Akt signaling pathway', 'Focal adhesion', 'Pathways in cancer', 'Bacterial invasion of epithelial cells' and 'Small cell lung cancer' (Fig. 2C). As for the medium dosage group (Fig. 2D), the DEGs were mainly enriched in 'PI3K-Akt signaling pathway', 'Pancreatic cancer',
'Sphingolipid signaling pathway', 'Pathways in cancer' and 'Proteoglycans in cancer'.

PPI network construction, module analysis and hub genes identification. As presented in Fig. 3A and B, the final PPI network of DEGs in the low dosage group was composed of 16 nodes and 22 edges after excluding the isolated nodes, whilst the PPI in the medium group contained 85 nodes and 201 edges. MCODE was used to analyze the key module (composed of genes with a relatively high degree of association) that consisted of five nodes and seven edges in the low dosage group (Fig. 3C) and two key modules in the medium dosage group (Fig. 3D). Module 1 consisted of nine nodes and 34 edges, where the genes were enriched in the KEGG pathway, including 'Pancreatic cancer', 'Central carbon metabolism in cancer' and 'Glioma' (data not shown). Module 2 consisted of six nodes and nine edges, where the genes were enriched in the KEGG pathways, including 'ECM-receptor interaction', 'Focal adhesion' and 'PI3K-Akt signaling pathway' (data not shown).

Salvianolate attenuates xenograft tumors in nude mice. Animal models were used to verify the effect of salvianolate on lung adenocarcinoma in vivo. In the present study, the maximum tumor diameter exhibited by any nude mouse was $10.8 \mathrm{~mm}$ and the specific data on the tumor volume and diameter over the entire experimental time period were shown in Table SI. The data in the present study revealed that salvianolate significantly reduced the tumor volume at 
Table II. GO functional enrichment and KEGG and Genomes pathway analysis of overlapping genes in the low and medium dosage groups.

\begin{tabular}{|c|c|c|c|}
\hline Gene symbol & Category & ID & Description \\
\hline \multirow[t]{7}{*}{ ARFIP2 } & BP & GO:0006928 & Movement of cell or subcellular component \\
\hline & $\mathrm{BP}$ & GO:0007264 & Small GTPase mediated signal transduction \\
\hline & $\mathrm{CC}$ & GO:0001726 & Ruffle \\
\hline & $\mathrm{CC}$ & GO:0005737 & Cytoplasm \\
\hline & MF & GO:0005515 & Protein binding \\
\hline & MF & GO:0005525 & GTP binding \\
\hline & KEGG_PATHWAY & 1 & 1 \\
\hline \multirow[t]{7}{*}{ CXCL6 } & $\mathrm{BP}$ & GO:0002446 & Neutrophil mediated immunity \\
\hline & $\mathrm{BP}$ & GO:0002690 & Positive regulation of leukocyte chemotaxis \\
\hline & $\mathrm{CC}$ & GO:0005576 & Extracellular region \\
\hline & $\mathrm{CC}$ & GO:0005615 & Extracellular space \\
\hline & MF & GO:0008009 & Chemokine activity \\
\hline & MF & GO:0008201 & Heparin binding \\
\hline & KEGG_PATHWAY & hsa04060 & Cytokine-cytokine receptor interaction \\
\hline \multirow[t]{7}{*}{ SHB } & $\mathrm{BP}$ & GO:0001525 & Angiogenesis \\
\hline & $\mathrm{BP}$ & GO:0006469 & Negative regulation of protein kinase activity \\
\hline & $\mathrm{CC}$ & GO:0005829 & Cytosol \\
\hline & $\mathrm{CC}$ & GO:0005886 & Plasma membrane \\
\hline & MF & GO:0001948 & Glycoprotein binding \\
\hline & MF & GO:0005070 & $\mathrm{SH} 3 / \mathrm{SH} 2$ adaptor activity \\
\hline & KEGG_PATHWAY & 1 & 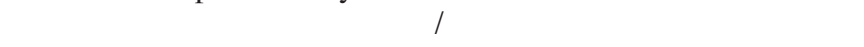 \\
\hline \multirow[t]{7}{*}{ SHC1 } & $\mathrm{BP}$ & GO:0000165 & MAPK cascade \\
\hline & BP & GO:0000187 & Activation of MAPK activity \\
\hline & $\mathrm{CC}$ & GO:0005622 & Intracellular \\
\hline & $\mathrm{CC}$ & GO:0005759 & Mitochondrial matrix \\
\hline & MF & GO:0005068 & Transmembrane receptor protein tyrosine kinase adaptor activity \\
\hline & MF & GO:0005088 & Ras guanyl-nucleotide exchange factor activity \\
\hline & KEGG_PATHWAY & hsa04012 & Erbb signaling pathway \\
\hline \multirow[t]{6}{*}{ COL6A1 } & $\mathrm{BP}$ & GO:0001649 & Osteoblast differentiation \\
\hline & $\mathrm{BP}$ & GO:0007155 & Cell adhesion \\
\hline & $\mathrm{CC}$ & GO:0005576 & Extracellular region \\
\hline & $\mathrm{CC}$ & GO:0005581 & Collagen trimer \\
\hline & MF & GO:0048407 & Platelet-derived growth factor binding \\
\hline & KEGG_PATHWAY & hsa04151 & PI3K-Akt signaling pathway \\
\hline \multirow[t]{7}{*}{ ELN } & $\mathrm{BP}$ & GO:0007519 & Skeletal muscle tissue development \\
\hline & BP & GO:0007585 & Respiratory gaseous exchange \\
\hline & $\mathrm{CC}$ & GO:0005576 & Extracellular region \\
\hline & $\mathrm{CC}$ & GO:0005578 & Proteinaceous extracellular matrix \\
\hline & MF & GO:0005201 & Extracellular matrix structural constituent \\
\hline & MF & GO:0005515 & Protein binding \\
\hline & KEGG_PATHWAY & hsa04974 & Protein digestion and absorption \\
\hline \multirow[t]{7}{*}{ ITGA2 } & $\mathrm{BP}$ & GO:0001666 & Response to hypoxia \\
\hline & $\mathrm{BP}$ & GO:0002687 & Positive regulation of leukocyte migration \\
\hline & $\mathrm{CC}$ & GO:0005634 & Nucleus \\
\hline & $\mathrm{CC}$ & GO:0005886 & Plasma membrane \\
\hline & MF & GO:0001618 & Virus receptor activity \\
\hline & MF & GO:0005178 & Integrin binding \\
\hline & KEGG_PATHWAY & hsa04145 & Phagosome \\
\hline \multirow[t]{7}{*}{ MAN1B1 } & $\mathrm{BP}$ & GO:0006491 & $\mathrm{N}$-glycan processing \\
\hline & BP & GO:0008152 & Metabolic process \\
\hline & $\mathrm{CC}$ & GO:0005783 & Endoplasmic reticulum \\
\hline & $\mathrm{CC}$ & GO:0005789 & Endoplasmic reticulum membrane \\
\hline & MF & GO:0004559 & Alpha-mannosidase activity \\
\hline & MF & GO:0004571 & Mannosyl-oligosaccharide 1,2-alpha-mannosidase activity \\
\hline & KEGG_PATHWAY & hsa00510 & $\mathrm{N}-$ Glycan biosynthesis \\
\hline \multirow[t]{4}{*}{ SAMD9L } & $\mathrm{BP}$ & GO:0034058 & Endosomal vesicle fusion \\
\hline & $\mathrm{CC}$ & GO:0005769 & Early endosome \\
\hline & MF & 1 & 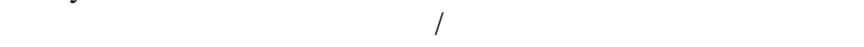 \\
\hline & KEGG_PATHWAY & / & l \\
\hline
\end{tabular}


A

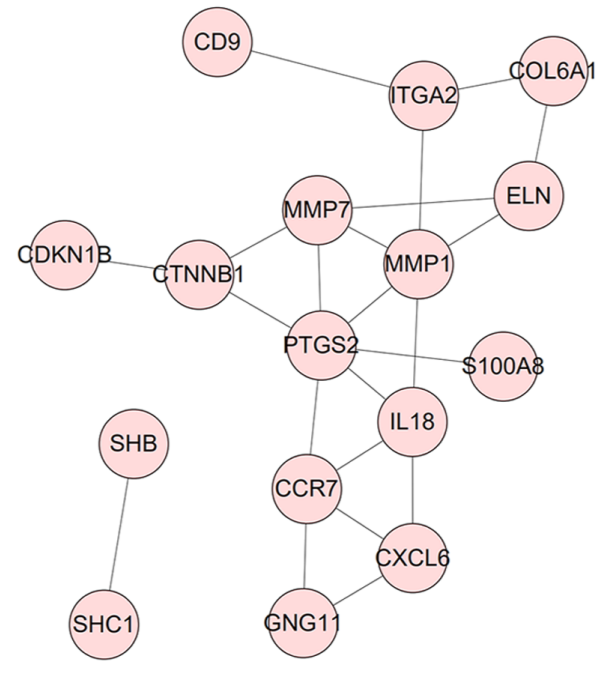

C

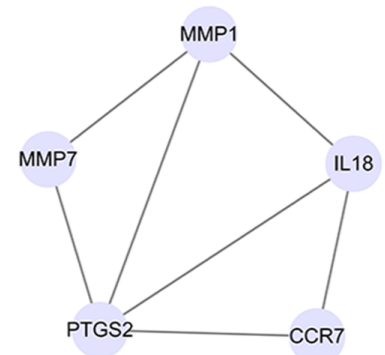

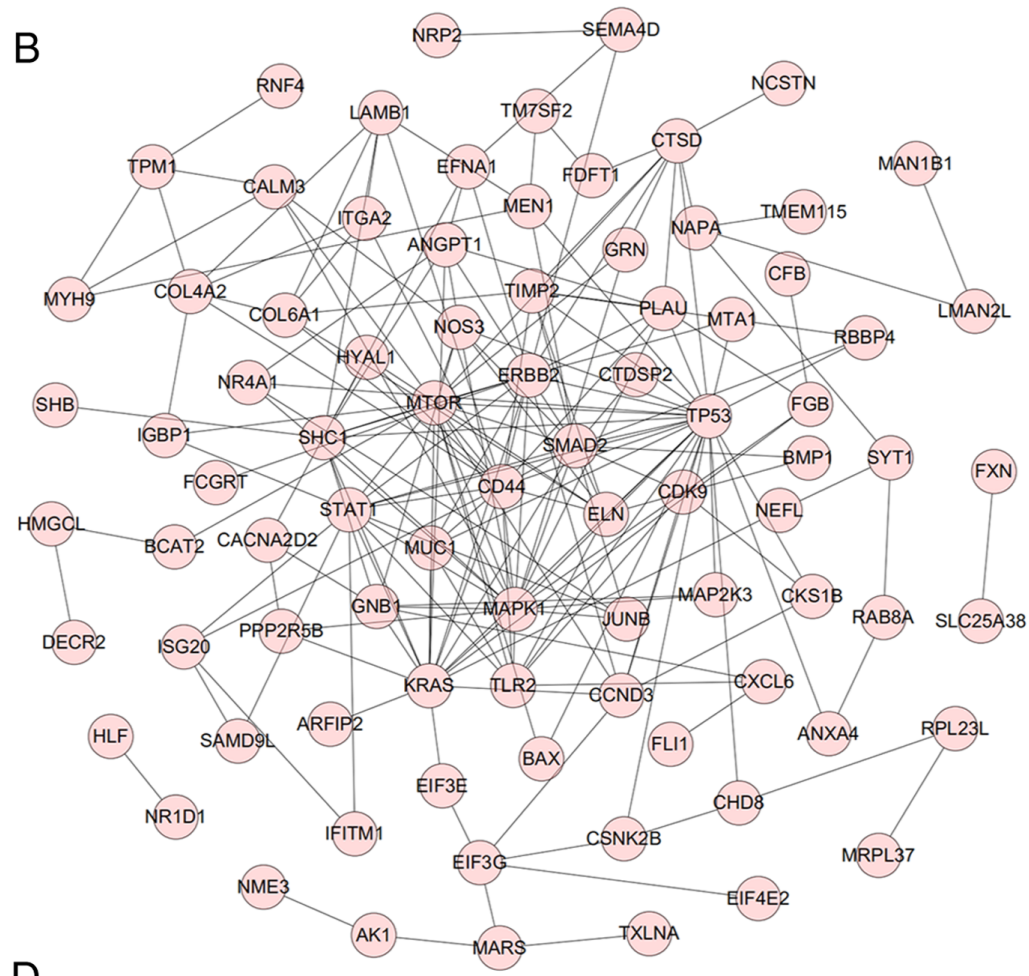

D

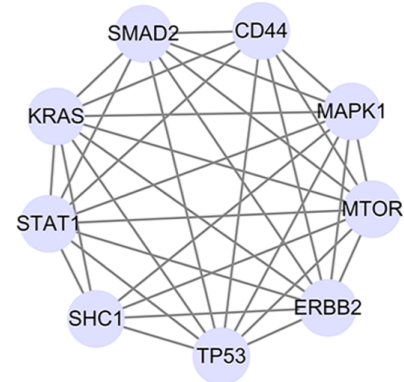

Module 1

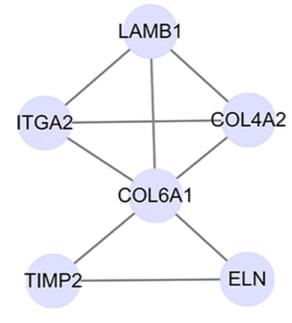

Module 2

Figure 3. PPI network and module analysis in the different dosage groups. PPI network of differentially expressed genes in the (A) low dosage and (B) medium dosage group were constructed using the Search Tool for the Retrieval of Interacting Genes/Proteins database. (C) A key module in the low dosage group and (D) two modules in the medium dosage group were identified using MCODE in the Cytoscape software. PPI, protein-protein interaction.

10,13 and 16 days, compared with that in the control group $(\mathrm{P}<0.05$; Fig. 4A). In addition, the inhibition rate of salvianolate on tumors was significantly increased in a dose-dependent manner (Fig. 4B). H\&E staining showed that the salvianolate administration groups exhibited nuclear pyknosis and fragmentation, suggesting apoptosis, compared with that in the control group (Fig. 4C).

Salvianolate treatment downregulates the expression of $A T P 7 A / 7 B$. IHC staining was performed to detect the expression levels of ATP7A and ATP7B. IHC showed that salvianolate significantly decreased the expression levels of ATP7A and ATP7B (Fig. 5A and B).

\section{Discussion}

Salvia miltiorrhiza has been applied as a TCM since it has been reported to possess anticancer properties (11). Components of Salvia miltiorrhiza can be divided into fat-soluble compounds that are comprised of diterpenoid quinones and water-soluble compounds, such as hydrophilic phenolic acids (13). The present study mainly investigated the effects of Tanshinone I (a diterpenoid quinones compound) and salvianolate (a water-soluble compound) on lung adenocarcinoma.

Tanshinone I is a notable component of the fat-soluble compounds that can be isolated from Salvia miltiorrhiza (28). In the present study, hub genes and key pathways involved in lung adenocarcinoma following administration of different dosages of Tanshinone I were identified. In the low dosage group (0.01 and $0.10 \mu \mathrm{g} / \mathrm{m}$ Tanshinone I vs. control group) and the medium dosage group (1 and $10 \mu \mathrm{g} / \mathrm{ml}$ Tanshinone I vs. control group), 28 DEGs and 58 DEGs were identified, respectively. Notably, the KEGG pathway analysis indicated that DEGs in the two groups were both enriched in the PI3K/Akt signaling pathway. Previous studies showed that Tanshinone I induced apoptosis by inhibiting the PI3K/Akt/mTOR pathway in ovarian cancer cells (29) and human breast cancer cell lines (30). Although there is some evidence to suggest that Tanshinone I can inhibit the proliferation of lung adenocarcinoma cell lines A549, CL1-0 and CL1-5 (14,22), further in vitro experiments 

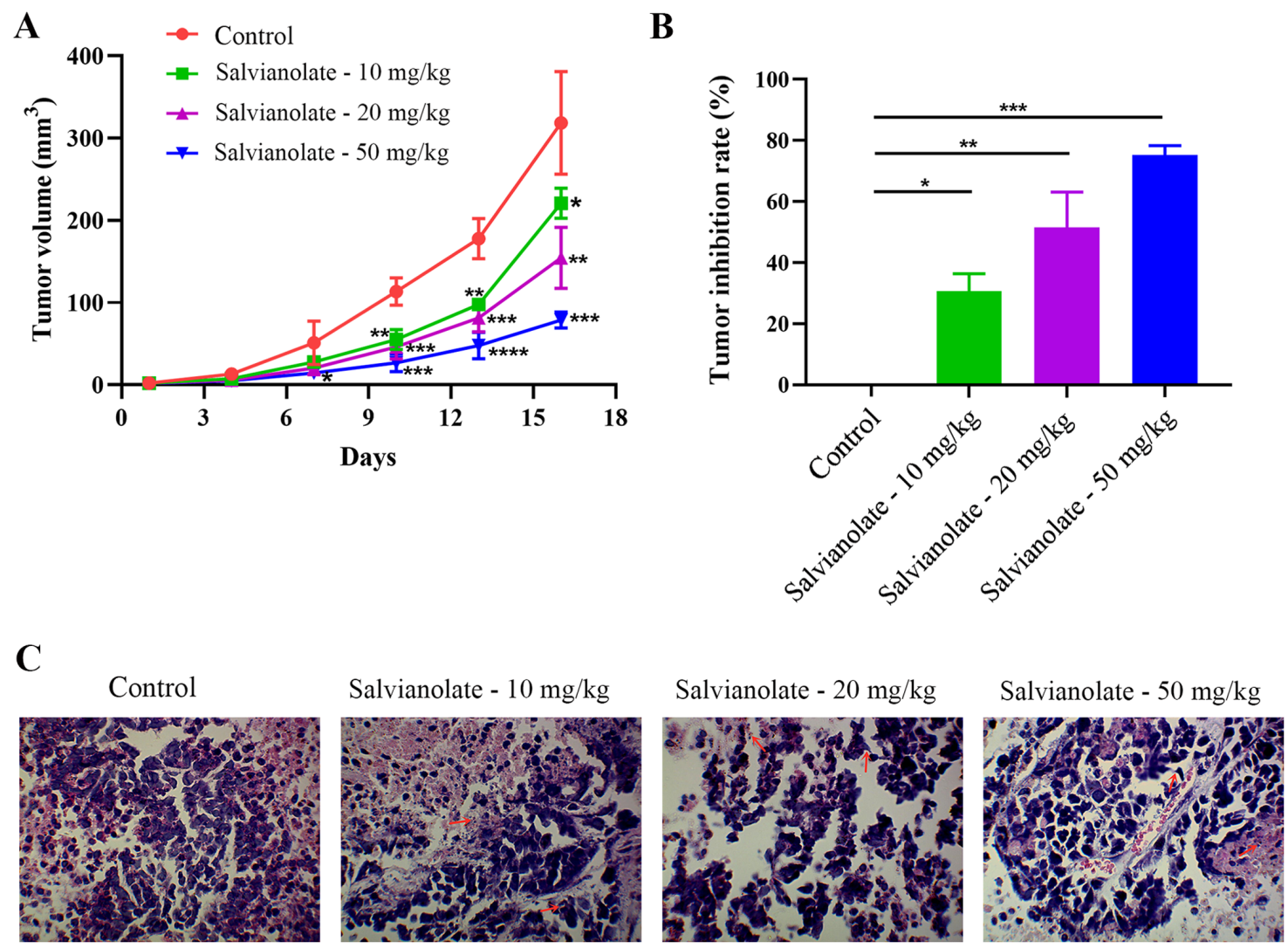

Figure 4. Salvianolate suppresses lung carcinoma xenograft tumor growth. (A) Compared with the control group, salvianolate significantly restricted the growth of tumors 10,13 and 16 days. (B) All three dosages of salvianolate increased the tumor inhibition rate, among which salvianolate (50 mg/kg) conferred the highest inhibition rate. (C) The tumor sections in the various groups were analyzed using hematoxylin and eosin staining (magnification, $\mathrm{x} 400$ ). Red arrows indicate nuclear pyknosis and fragmentation. ${ }^{*} \mathrm{P}<0.05,{ }^{* *} \mathrm{P}<0.01,{ }^{* * *} \mathrm{P}<0.001$ and ${ }^{* * * * *} \mathrm{P}<0.0001$ vs. Control group.

A

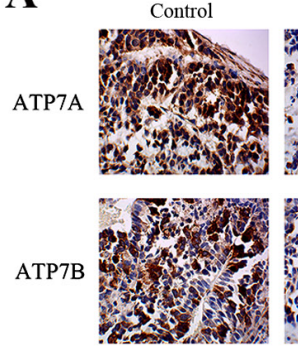

$10 \mathrm{mg} / \mathrm{kg}$
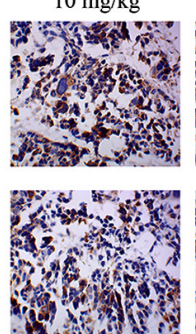

$20 \mathrm{mg} / \mathrm{kg}$

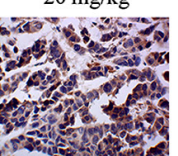

$50 \mathrm{mg} / \mathrm{kg}$

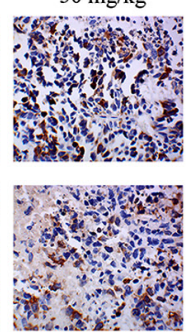

B

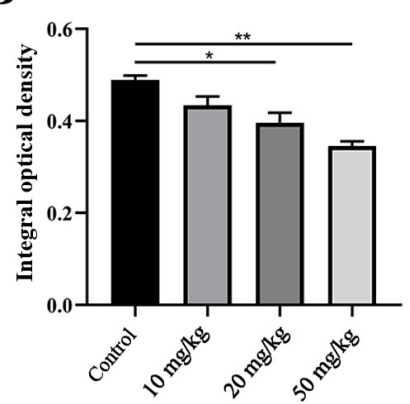

ATP7B

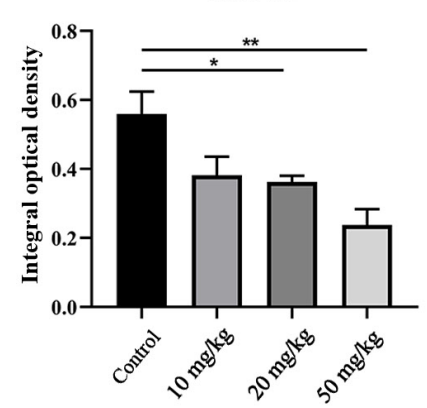

Figure 5. Expression levels of ATP7A and ATP7B are decreased following salvianolate administration. (A) Representative images of immunohistochemical staining for ATP7A and ATP7B expression on tumor tissues from the nude mice (magnification, x400). (B) Integral optical densities of ATP7A and ATP7B were analyzed. ${ }^{*} \mathrm{P}<0.05$ and ${ }^{* *} \mathrm{P}<0.01$. ATP7, ATPase copper transporting.

are required to investigate the underlying mechanism and molecular target of Tanshinone I in lung adenocarcinoma.

Notably, nine overlapping DEGs were identified in the low and medium dosage groups, which warrant further study. ITGA 2 is the $\alpha$ subunit of a transmembrane receptor that has been reported to regulate cell migration and differentiation (31). Previous studies have shown that ITGA2 expression was upregulated in several different types of cancer (32), including gastric (33) and breast cancer (34). The present study indicated that ITGA2 expression was downregulated by Tanshinone I in lung adenocarcinoma, which may be a potential target. MAN1B1 encodes $\alpha$-1,2-mannosidase, which mediates protein glycosylation modification and glycoprotein polysaccharide hydrolysis (35). It has been reported that high expression levels of MAN1B1 were associated with poor prognosis in bladder cancer (36). However, the effects of MAN1B1 downregulation induced by Tanshinone I on responses in lung adenocarcinoma require further investigation. Other studies have shown that SHB knockdown increased the susceptibility of the SVR angiosarcoma cell line to cisplatin and staurosporine (37) whilst impairing the growth of tumors in mice injected with Lewis lung carcinoma cells or T241 fibrosarcoma cells (38). The present study demonstrated the association between Tanshinone I treatment and the downregulation of SHB in 
lung adenocarcinoma. ELN is a signature extracellular matrix protein in the lungs, where ELN-derived fragments have been shown to be pro-tumorigenic (39). The SHC1 protein exists in three isoforms, p46SHC, p52SHC and p66SHC (40). $\mathrm{Xu}$ et al (41) demonstrated that salvianolic acid A pretreatment increased the expression of sirtuin 1, which was associated with downregulation of p66SHC, a growth factor adapter $\mathrm{SHC}$, in a drug-induced liver injury mouse model. COL6A1, which contributes to maintaining the integrity of tissues, was found to be upregulated in cervical cancer (42) and pancreatic cancer tissues (43) compared with that in adjacent non-tumor tissues. It has been reported that overexpression of ARFIP2 inhibited tumor necrosis factor- $\alpha$-stimulated $N F-\kappa B$ signaling by interacting with inhibitor of $N F-\kappa B$ kinase $\beta / N F-\kappa B$ essential modulator (44). CXCL6 is a chemokine that participates in cancer angiogenesis, metastasis and the immune response (45), where it has been reported to promote NSCLC cell survival and metastasis (46). CXCL6 expression was demonstrated to be upregulated by Tanshinone I treatment in a lung adenocarcinoma cell line in the present study. SAMD9L had been demonstrated to be a tumor suppressor in breast, hepatocellular and squamous cell carcinoma (47). However, its functional role remains poorly understood. In the present study, bioinformatics analysis revealed that these nine genes were DEGs following the administration of different doses of Tanshinone I, the mechanistic details of which warrants further investigation.

Salvianolate is comprised of salvianolic acids A and B and is a major water-soluble component in the extract of Salvia miltiorrhiza. The present study showed that salvianolate significantly decreased the tumor volumes of xenograft nude mice, compared with those in the control group. It would therefore be useful to investigate if Tanshinone I and salvianolate exert synergistic effects on lung adenocarcinoma in future studies. Although the IHC expression of ATP7A in $20 \mathrm{mg} / \mathrm{kg}$ salvianolate appeared to be higher compared with that in $10 \mathrm{mg} / \mathrm{kg}$, it can still be concluded that, overall, salvianolate (10, 20 and $50 \mathrm{mg} / \mathrm{kg}$ ) decreased the expression of ATP7A and ATP7B in a concentration-dependent manner. ATP7A/7B are copper efflux transporters that maintain cellular copper homeostasis (15). Whether copper homeostasis or other pathways are part of the underlying mechanism mediated by the treatment of salvianolate remains to be investigated.

To conclude, the present study revealed the DEGs and underlying pathways in lung adenocarcinoma following treatment with different doses of Tanshinone I using bioinformatics tools. In addition, the present study also verified the antitumor effects of salvianolate, another active ingredient of Salvia miltiorrhiza, in animals and lung adenocarcinoma cells. These findings suggest the potential value of applying the Salvia miltiorrhiza extract for lung adenocarcinoma treatment.

\section{Acknowledgements}

Not applicable.

\section{Funding}

The present study was financially supported by the Hunan Provincial Natural Science Foundation of China (grant no. 2019JJ40519) and Hunan Province Traditional Chinese Medicine Research Project (grant no. 201179).

\section{Availability of data and materials}

The datasets used and/or analyzed during the current study are available from the corresponding author on reasonable request.

\section{Authors' contributions}

HT, YL, JC and XL conceived and designed the study. HT and JM performed animal experiments and collected data. HT, YL and LC, JC and XL analyzed and interpreted the data. JC and $\mathrm{XL}$ revised the manuscript. JY and ZL interpreted the data and checked the revised version of the manuscript. All authors read and approved the manuscript.

\section{Ethics approval and consent to participate}

All animal research protocols were following with the guidelines of the Ethics Committee of Institutional Animal Care and Use in Central South University and approved by Medical Ethics Committee of Xiangya Hospital, Central South University (approval no. 2017121170; Changsha, China).

\section{Patient consent for publication}

Not applicable.

\section{Competing interests}

The authors declare that they have no competing interests.

\section{References}

1. Barta JA, Powell CA and Wisnivesky JP: Global epidemiology of lung cancer. Ann Glob Health 85: 85, 2019.

2. World Health Organization: Cancer Today. 2021. Accessed from https://gco.iarc.fr/today/home.

3. Blandin Knight S, Crosbie PA, Balata H, Chudziak J, Hussell T and Dive C: Progress and prospects of early detection in lung cancer. Open Biol 7: 7, 2017.

4. Herbst RS, Morgensztern D and Boshoff C: The biology and management of non-small cell lung cancer. Nature 553: 446-454, 2018.

5. Zhou K,Zhao S, GuoW and Ding L: Efficacy and safety of erlotinib combined with bevacizumab in the treatment of non-small cell lung cancer: A systematic review and meta-analysis. Medicine (Baltimore) 99: e18771, 2020.

6. Atal S, Asokan P and Jhaj R: Recent advances in targeted small-molecule inhibitor therapy for non-small-cell lung cancer-An update. J Clin Pharm Ther 45: 580-584, 2020.

7. Zugazagoitia J, Guedes C, Ponce S, Ferrer I, Molina-Pinelo S and Paz-Ares L: Current challenges in cancer treatment. Clin Ther 38: 1551-1566, 2016.

8. Liao YH, Li CI, Lin CC, Lin JG, Chiang JH and Li TC: Traditional Chinese medicine as adjunctive therapy improves the long-term survival of lung cancer patients. J Cancer Res Clin Oncol 143: 2425-2435, 2017.

9. Wang Y, Liu Y, Du X, Ma H and Yao J: The anti-cancer mechanisms of berberine: A Review. Cancer Manag Res 12: 695-702, 2020.

10. Guo Q, Li J and Lin H: Effect and molecular mechanisms of traditional Chinese medicine on regulating tumor immunosuppressive microenvironment. BioMed Res Int 2015: 261620, 2015.

11. Lin YY, Lee IY, Huang WS, Lin YS, Kuan FC, Shu LH, Cheng YC, Yang YH and Wu CY: Danshen improves survival of patients with colon cancer and dihydroisotanshinone I inhibit the proliferation of colon cancer cells via apoptosis and skp2 signaling pathway. J Ethnopharmacol 209: 305-316, 2017. 
12. Lin YS, Shen YC, Wu CY, Tsai YY, Yang YH, Lin YY, Kuan FC, Lu CN, Chang GH, Tsai MS, et al: Danshen Improves Survival of Patients With Breast Cancer and Dihydroisotanshinone I Induces Ferroptosis and Apoptosis of Breast Cancer Cells. Front Pharmacol 10: 1226, 2019.

13. Su CY, Ming QL, Rahman K, Han T and Qin LP: Salvia miltiorrhiza: Traditional medicinal uses, chemistry, and pharmacology. Chin J Nat Med 13: 163-182, 2015.

14. Tung YT, Chen HL, Lee CY, Chou YC, Lee PY, Tsai HC, Lin YL and Chen CM: Active component of Danshen (Salvia miltiorrhiza Bunge), tanshinone I, attenuates lung tumorigenesis via inhibitions of VEGF, Cyclin A, and Cyclin B expressions. Evid Based Complement Alternat Med 2013: 319247, 2013.

15. La Fontaine S and Mercer JF: Trafficking of the copper-ATPases, ATP7A and ATP7B: Role in copper homeostasis. Arch Biochem Biophys 463: 149-167, 2007.

16. Shanbhag V, Jasmer-McDonald K, Zhu S, Martin AL, Gudekar N, Khan A, Ladomersky E, Singh K, Weisman GA and Petris MJ: ATP7A delivers copper to the lysyl oxidase family of enzymes and promotes tumorigenesis and metastasis. Proc Natl Acad Sci USA 116: 6836-6841, 2019.

17. Yang T, Chen M, Chen T and Thakur A: Expression of the copper transporters hCtr1, ATP7A and ATP7B is associated with the response to chemotherapy and survival time in patients with resected non-small cell lung cancer. Oncol Lett 10: 2584-2590, 2015.

18. Li YQ, Chen J, Yin JY, Liu ZQ and Li XP: Gene expression and single nucleotide polymorphism of ATP7B are associated with platinum-based chemotherapy response in non-small cell lung cancer patients. J Cancer 9: 3532-3539, 2018.

19. Samimi G, Safaei R, Katano K, Holzer AK, Rochdi M, Tomioka M, Goodman M and Howell SB: Increased expression of the copper efflux transporter ATP7A mediates resistance to cisplatin, carboplatin, and oxaliplatin in ovarian cancer cells Clin Cancer Res 10: 4661-4669, 2004.

20. Li YQ, Zhang XY, Chen J, Yin JY and Li XP: ATP7B rs9535826 is associated with gastrointestinal toxicity of platinum-based chemotherapy in nonsmall cell lung cancer patients. J Cancer Res Ther 14: 881-886, 2018.

21. Li YQ, Yin JY, Liu ZQ and Li XP: Copper efflux transporters ATP7A and ATP7B: Novel biomarkers for platinum drug resistance and targets for therapy. IUBMB Life 70: 183-191, 2018.

22. Lee CY, Sher HF, Chen HW, Liu CC, Chen CH, Lin CS, Yang PC, Tsay HS and Chen JJ: Anticancer effects of tanshinone I in human non-small cell lung cancer. Mol Cancer Ther 7: 3527-3538, 2008

23. Huang W, Sherman BT and Lempicki RA: Systematic and integrative analysis of large gene lists using DAVID bioinformatics resources. Nat Protoc 4: 44-57, 2009.

24. Walter W, Sánchez-Cabo F and Ricote M: GOplot: An R package for visually combining expression data with functional analysis. Bioinformatics 31: 2912-2914, 2015.

25. Szklarczyk D, Franceschini A, Wyder S, Forslund K, Heller D, Huerta-Cepas J, Simonovic M, Roth A, Santos A, Tsafou KP, et al: STRING v10: Protein-protein interaction networks, integrated over the tree of life. Nucleic Acids Res 43: D447-D452, 2015.

26. Doncheva NT, Morris JH, Gorodkin J and Jensen LJ: Cytoscape StringApp: Network analysis and visualization of proteomics data. J Proteome Res 18: 623-632, 2019.

27. Ministry of Science and Technology of the People's Republic of China: 2021. Accessed from: http://www.most.gov.cn/xxgk/ xinxifenlei/fdzdgknr/fgzc/gfxwj/gfxwj2010before/201712/ t20171222_137025.html

28. Fu L, Han B, Zhou Y, Ren J, Cao W, Patel G, Kai G and Zhang J: The anticancer properties of tanshinones and the pharmacological effects of their active ingredients. Front Pharmacol 11: $193,2020$.

29. Zhou J, Jiang YY, Chen H, Wu YC and Zhang L: Tanshinone I attenuates the malignant biological properties of ovarian cancer by inducing apoptosis and autophagy via the inactivation of PI3K/AKT/mTOR pathway. Cell Prolif 53: e12739, 2020.

30. Wang L, Wu J, Lu J, Ma R, Sun D and Tang J: Regulation of the cell cycle and PI3K/Akt/mTOR signaling pathway by tanshinone I in human breast cancer cell lines. Mol Med Rep 11: 931-939, 2015.
31. Adorno-Cruz $\mathrm{V}$ and Liu $\mathrm{H}$ : Regulation and functions of integrin $\alpha 2$ in cell adhesion and disease. Genes Dis 6: 16-24, 2018.

32. Ren D, Zhao J, Sun Y, Li D, Meng Z, Wang B, Fan P, Liu Z, Jin $\mathrm{X}$ and $\mathrm{Wu} \mathrm{H}$ : Overexpressed ITGA2 promotes malignant tumor aggression by up-regulating PD-L1 expression through the activation of the STAT3 signaling pathway. J Exp Clin Cancer Res 38: 485, 2019.

33. Chuang YC, Wu HY, Lin YL, Tzou SC, Chuang CH, Jian TY, Chen PR, Chang YC, Lin CH, Huang TH, et al: Blockade of ITGA2 Induces Apoptosis and Inhibits Cell Migration in Gastric Cancer. Biol Proced Online 20: 10, 2018.

34. Ding W, Fan XL, Xu X, Huang JZ, Xu SH, Geng Q, Li R, Chen D and Yan GR: Epigenetic silencing of ITGA 2 by MiR-373 promotes cell migration in breast cancer. PLoS One 10: e0135128, 2015.

35. Van Scherpenzeel M, Timal S, Rymen D, Hoischen A, Wuhrer M, Hipgrave-Ederveen A, Grunewald S, Peanne R, Saada A, Edvardson $\mathrm{S}$, et al: Diagnostic serum glycosylation profile in patients with intellectual disability as a result of MAN1B1 deficiency. Brain 137: 1030-1038, 2014.

36. Wang HF, Wu JH, Gai JW, Yang SQ, Ma QT, Ma HS and Feng Q: MAN1B1 is associated with poor prognosis and modulates proliferation and apoptosis in bladder cancer. Gene 679: 314-319, 2018.

37. Funa NS, Reddy K, Bhandarkar S, Kurenova EV, Yang L, Cance WG, Welsh M and Arbiser JL: Shb gene knockdown increases the susceptibility of SVR endothelial tumor cells to apoptotic stimuli in vitro and in vivo. J Invest Dermatol 128: 710-716, 2008.

38. Funa NS, Kriz V, Zang G, Calounova G, Akerblom B, Mares J, Larsson E, Sun Y, Betsholtz C and Welsh M: Dysfunctional microvasculature as a consequence of shb gene inactivation causes impaired tumor growth. Cancer Res 69: 2141-2148, 2009.

39. Thorlacius-Ussing J, Kehlet SN, Rønnow SR, Karsdal MA and Willumsen N: Non-invasive profiling of protease-specific elastin turnover in lung cancer: Biomarker potential. J Cancer Res Clin Oncol 145: 383-392, 2019

40. Wright KD, Miller BS, El-Meanawy S, Tsaih SW, Banerjee A, Geurts AM, Sheinin Y, Sun Y, Kalyanaraman B, Rui H, et al: The p52 isoform of SHC1 is a key driver of breast cancer initiation. Breast Cancer Res 21: 74, 2019.

41. Xu X, Hu Y, Zhai X, Lin M, Chen Z, Tian X, Zhang F, Gao D, Ma X, Lv L, et al: Salvianolic acid A preconditioning confers protection against concanavalin A-induced liver injury through SIRT1-mediated repression of p66shc in mice. Toxicol Appl Pharmacol 273: 68-76, 2013.

42. Hou T, Tong C, Kazobinka G, Zhang W, Huang X, Huang Y and Zhang Y: Expression of COL6A1 predicts prognosis in cervical cancer patients. Am J Transl Res 8: 2838-2844, 2016.

43. Owusu-Ansah KG, Song G, Chen R, Edoo MIA, Li J, Chen B, Wu J, Zhou L, Xie H, Jiang D, et al: COL6A1 promotes metastasis and predicts poor prognosis in patients with pancreatic cancer. Int J Oncol 55: 391-404, 2019.

44. You DJ, Park CR, Furlong M, Koo O, Lee C, Ahn C, Seong JY and Hwang JI: Dimer of arfaptin 2 regulates $N F-\kappa B$ signaling by interacting with IKK $\beta / \mathrm{NEMO}$ and inhibiting IKK $\beta$ kinase activity. Cell Signal 27: 2173-2181, 2015.

45. Verbeke H, Struyf S, Berghmans N, Van Coillie E, Opdenakker G, Uyttenhove C, Van Snick J and Van Damme J: Isotypic neutralizing antibodies against mouse GCP-2/CXCL6 inhibit melanoma growth and metastasis. Cancer Lett 302: 54-62, 2011.

46. Li J, Tang Z, Wang H, Wu W, Zhou F, Ke H, Lu W, Zhang S, Zhang Y, Yang S, et al: CXCL6 promotes non-small cell lung cancer cell survival and metastasis via down-regulation of miR-515-5p. Biomed Pharmacother 97: 1182-1188, 2018.

47. Davidsson J, Puschmann A, Tedgård U, Bryder D, Nilsson L and Cammenga J: SAMD9 and SAMD9L in inherited predisposition to ataxia, pancytopenia, and myeloid malignancies. Leukemia 32: 1106-1115, 2018.

This work is licensed under a Creative Commons Attribution-NonCommercial-NoDerivatives 4.0 International (CC BY-NC-ND 4.0) License. 